\title{
Comment to the letter by Drs. Safiri and Ashrafi-Asgarabad
}

\author{
Yaakov Melcer ${ }^{1,2} \cdot$ Ron Maymon $^{1,2} \cdot$ Marina Pekar-Zlotin ${ }^{1,2} \cdot$ Zvi Vaknin $^{1,2} \cdot$ Moty Pansky $^{1,2} \cdot$ Noam Smorgick $^{1,2}$
}

Received: 6 February 2018 / Accepted: 28 March 2018 / Published online: 9 March 2018

c) Springer-Verlag GmbH Germany, part of Springer Nature 2018

Dear Editor,

We thank Drs. Safiri and Ashrafi-Asgarabad for their questions and comments.

We agree with Drs. Safiri and Ashrafi-Asgarabad that relatively rare signs and symptoms, such as peritoneal irritation signs in women presenting with adnexal torsion, may be problematic for statistical interpretation and that more sophisticated statistical methods suggested by Drs. Safiri and Ashrafi-Asgarabad could be more appropriate.

However, the finding of positive peritoneal irritation signs in women with suspected adnexal torsion is only one of several clinical, laboratory and ultrasound parameters described in our study, and by no means the most important one. Because the diagnosis of adnexal torsion is often difficult, we emphasize in our study that all of these parameters should be assessed in women with suspected torsion, rather than relying on a single finding on the physical examination.

\section{Compliance with ethical standards}

Conflict of interest The authors declare that they have no conflict of interests.
Yaakov Melcer

ymeltcer@gmail.com

1 Department of Obstetrics and Gynecology, Assaf Harofeh Medical Center, 70300 Zerifin, Israel

2 Affiliated with the Tel-Aviv University, Sackler School of Medicine, Tel-Aviv, Israel 